\title{
Species composition and distribution of decapod crustaceans in the waters off Patagonia and Tierra del Fuego, South America*
}

\author{
WOLF E. ARNTZ1 ${ }^{1}$ MATTHIAS GORNY², RAUL SOTO ${ }^{3}$, MARCO A. LARDIES ${ }^{4}$, \\ MARCO RETAMAL ${ }^{5}$ and INGO S. WEHRTMANN ${ }^{1,6}$ \\ ${ }^{1}$ Alfred-Wegener-Institut für Polar- und Meeresforschung, Bremerhaven, Germany. \\ ${ }^{2}$ Instituto de la Patagonia, Universidad de Magallanes, Punta Arenas, Chile. \\ ${ }^{3}$ Dpto. de Oceanografía, Universidad Arturo Prat, Iquique, Chile. \\ ${ }^{4}$ Instituto de Biología Marina "Jürgen Winter", Univ. Austral de Chile, Valdivia, Chile. \\ ${ }^{5}$ Dpto. de Oceanografía, Universidad Concepción, Concepción, Chile. \\ ${ }^{6}$ Instituto de Zoología “Ernst F. Kilian”, Univ. Austral de Chile, Valdivia, Chile.
}

\begin{abstract}
SUMMARY: This paper comprises the decapod crustacean material collected during the "Victor Hensen" campaign 1994 and during the last phase of "Polarstern" cruise ANT XIII/4 in 1996. Sampling covered waters of medium and greater depth in the Straits of Magellan, the channels towards the south, and the area south of the Beagle Channel including the northern slope of the Drake Passage. 30 species of decapods were found, which is considered a good result taking into account that the species restricted to the intertidal and the upper subtidal, which were not subject of this study, are missing. Anomurans were the dominant group, followed by brachyurans and caridean shrimps. Additionally one palinuran (Stereomastis suhmi; $\mathrm{n}=8$ ) and a single specimen of the astacuran Thymops birsteini were found. Dominance patterns changed considerably from the Straits of Magellan to the channel system south of the Strait, and again to the area south of the Beagle and the northern slope of the Drake Passage. Among the top dominants were the galatheid Munida spp., which in the southernmost area numerically contributes $>90 \%$ to the catches, the brachyuran crabs Peltarion spinosulum and Eurypodius latreillii, the hermit crab Pagurus comptus, and the caridean shrimps Austropandalus grayi and Pasiphaea acutifrons. Munida spp. and $P$. spinosulum also revealed the highest frequency of occurrence at $49 \%$ and $45 \%$ of the stations, respectively, followed by $E$. latreillii, P. comptus, and A. grayi. Regional and bathymetric distribution of the principal species are given. The depth range of some species was found to be very wide (Campylonotus semistriatus, Munida spp., E. latreillii, P. spinosulum) whereas others were restricted to relatively shallow waters (Pagurus gaudichaudii, Lithodes santolla, Campylonotus vagans, A. grayi) or great depth (Nematocarcinus lanceopes, Stereomastis suhmi). It should be mentioned, however, that sampling depths differed greatly in the areas of study. Contrary to the Antarctic, the Magellan region reveals a strong dominance of reptant decapods whereas caridean shrimps play a minor role in that region. This dominance pattern is valid even for the southernmost areas of the region. The northern slope of the Drake Passage is characterized by a mixture of cold-temperate and Antarctic elements which make it a transitional area between the two continents.
\end{abstract}

Key words: Decapoda, species composition, distribution, abundance, Straits of Magellan, Beagle Channel, Drake Passage

RESUMEN: COMPOSICIÓN DE ESPECIES Y DISTRIBUCIÓN DE LOS CRUSTÁCEOS DECÁPODOS EN LAS AGUAS DE LA PATAGONIA Y DE TierRa DEl Fuego, AmÉricA DEL SuR. - En este trabajo se analiza el material de crustáceos decápodos recolectado en la Campaña "Victor Hensen" 1994 y en la última etapa de la campaña "Polarstern" ANT XIII/4 en 1996. El muestreo cubrió las aguas de mediana y mayor profundidad en el Estrecho de Magallanes, los canales del sur y el área al sur del Canal del Beagle incluyendo la vertiente norte del Paso de Drake. Se registraron 30 especies de decápodos, lo cual se considera un número apreciable, teniendo en cuenta que no se incluyen las especies del intermareal y del infralitoral somero, que no fueron objeto de estudio en este trabajo. Los anomuros fueron el grupo predominante, seguidos por los braquiuros y los carídeos. También se encontraron un palinúrido (Stereomastis suhmi), representado por 8 ejemplares, y el astacúrido Thymops 
birsteini con un solo ejemplar. Los patrones de dominancia cambiaron sustancialmente del Estrecho de Magallanes hacia el sistema de canales al sur del Estrecho, y otra vez hacia el área al sur del Beagle y la vertiente norte del Paso de Drake. Entre las especies predominantes figuraron los galateídeos Munida spp., que en el área más meridional contribuyó más del $90 \%$ de las capturas, los cangrejos braquiuros Peltarion spinosulum y Eurypodius latreillii, el cangrejo ermitaño Pagurus comptus y los camarones carídeos Austropandalus grayi y Pasiphaea acutifrons. Munida spp. and P. spinosulum presentaron también la mayor presencia con 49 y $45 \%$ de las estaciones respectivamente, seguidas por E. latreillii, P. comptus y A. grayi. Se presenta la distribución regional y batimétrica de las principales especies. El ámbito de profundidad de algunas especies $(C$. semistriatus, Munida spp., E. latreillii, $P$. spinosulum) fue muy amplio mientras que otras especies se restringieron a aguas relativamente someras (Pagurus gaudichaudii, Lithodes santolla, Campylonotus vagans, Austropandalus grayi) o profundas (Nematocarcinus lanceopes, Stereomastis suhmi). Hay que mencionar, sin embargo, que las profundidades de muestreo en las diferentes áreas fueron muy distintas. Al contrario que la Antártida, la región Magallánica revela una fuerte dominancia de los decápodos reptantes, mientras que los carídeos de esta región cumplen un papel de menor importancia en cuanto al porcentaje de captura. Esta dominancia es válida incluso para las partes más al sur de la región. La vertiente norte del Paso de Drake se caracteriza por una mezcla de elementos antárticos y de aguas templadas frías, lo que la convierte en un área de transición entre los dos continentes.

Palabras clave: Decapoda, composición de especies, distribución, abundancia, Estrecho de Magallanes, Canal del Beagle, Paso de Drake

\section{INTRODUCTION}

The distribution of decapod crustaceans in the numerous channels and fjords, which form the southern tip of the South American continent, was poorly known until some years ago. The principal source of information had been the Hamburger Magalhaensische Sammelreise (Doflein and Balss, 1912), with various records obtained in the southernmost part, around Navarino Island. During several other expeditions, among them the University of Lund Chile Expedition 1948-49 (Brattström and Dahl, 1951), which provided the more recent base of information on the Chilean decapod fauna (cf. Brattström and Johanssen, 1983), only few occasional samples were taken in the area between $42^{\circ}$ and $55^{\circ} \mathrm{S}$. Both the species inventory and the distribution of selected decapod groups have been actualized recently (Retamal and Soto 1993, 1995; Wehrtmann and Carvacho 1997), and Boschi et al. (1992) have summarized the present knowledge regarding the faunal composition and distribution of decapod crustaceans along the Atlantic Patagonian coast. However, these studies either focused on selected groups, or were restricted to certain sectors of our area of study.

With respect to Antarctic-South American comparisons, the decapod material obtained by the Soviet Antarctic expeditions between 1955 and 1958 was of particular interest, because the geographical scope of sampling covered not only the Antarctic but included also the waters off Chile and South New Zealand (Zarenkov, 1970). From the information available at that time, Zarenkov (loc.cit.) derived the existence of two antiboreal faunas, South American and New Zealand. Furthermore, he concluded that the connections of the decapod fauna on the Antarctic shelves with that living in areas north of the Antarctic Convergence are weak and confined to South America and the Subantarctic islands of Kerguelen and the Scotia Arc. However, information on the decapod fauna of the Subantarctic islands was sparse at the time, and Zarenkov's samples from southern South America were scattered over a comparatively large area. A more detailed data base served for the recent description of the biogeography of decapods in the Southern Ocean by Gorny (1999).

Species composition and distribution of decapods in the Antarctic have been the subject of more intense study during the past decade, revealing that this group is not as scarcely represented as was assumed two decades ago. Current species composition of the Antarctic decapod fauna is dominated by caridean shrimps and few lithodid anomuran crabs (e.g., Kirkwood, 1984; Arntz and Gorny, 1991; Klages et al., 1995; Gorny, 1999). However, the fossil record clearly documents that decapods were more abundant and diverse in the southernmost parts of the Southern Ocean during the Cretaceous and early Tertiary up to Eocene times (see Feldmann and Quilty, 1997 and references cited therein).

The purpose of the present study is to describe in more detail the species composition and distribution patterns between the Strait of Magellan and Cape Horn, considering possible trends (e.g., does species richness decrease with latitude?) and (dis)similarities with the Antarctic decapod fauna (e.g., is the northern slope of the Drake Passage a transitional area?). New biogeographical aspects that mainly emerged from the recent revision of the decapod fauna of the Southern Ocean by Gorny (1999) will be considered. 


\section{MATERIAL AND METHODS}

Samples were obtained during the Joint "Victor Hensen" Magellan Campaign (30 October-15 November 1994) and leg 4 of RV "Polarstern" cruise ANT XIII (14-18 May 1996). The study site (cf. Fig. 5) covered the areas between $52^{\circ} 56^{\prime} \mathrm{S}$, $70^{\circ} 18^{\prime} \mathrm{W}$ and $55^{\circ} \quad 47^{\prime} \mathrm{S}, 6^{\circ} 57^{\prime} \mathrm{W}$ ("Victor Hensen"), and between $55^{\circ} 23^{\prime} \mathrm{S}, 66^{\circ} 13^{\prime} \mathrm{W}$ and $55^{\circ} 47^{\prime} \mathrm{S}, 65^{\circ} 49^{\prime} \mathrm{W}$ ("Polar-stern”). Decapod crustaceans were collected by means of a modified Agassiz trawl (AGT) with a beam length of $1.5 \mathrm{~m}$ and $10 \mathrm{~mm}$ mesh size in the codend. A total of 72 successful hauls ("Victor Hensen": 63 hauls; "Polarstern": 9 hauls) were obtained and analyzed. Trawling depths of the successful AGT hauls during the "Victor Hensen" and "Polarstern" cruises varied from 9-653 $\mathrm{m}$ and 99-2505 $\mathrm{m}$, respectively. The average trawling depths (extremes in brackets) were $130 \mathrm{~m}$ (9-527) for the Straits of Magellan, $261 \mathrm{~m}$ (20-653) for the channels, and $50 \mathrm{~m}$ (15-112) viz. $915 \mathrm{~m}$ (98-2505) for the area south of the Beagle on "Victor Hensen" and "Polarstern", respectively. For further details see cruise reports by Arntz and Gorny (1996) and Fahrbach and Gerdes (1997).

Decapods were picked quantitatively from the total catch at nearly all stations. An exception were some extraordinarily large or repetitive (in terms of depth) hauls from stations VH 1203, 1209, 1215, 1223, 1225 and 1242 located south of the Beagle Channel (marked with an * in Table 1). From these hauls, the most abundant decapod Munida spp. was registered as "no. of baskets" only, using haul VH 1149 , where the number of specimens per basket was counted $(n=3000)$, as a reference station. Numbers of the two most abundant brachyuran species, Peltarion spinosulum and Eurypodius latreillii, also had to be backcalculated for stations 1215, 1223, 1225, 1242 and 1223, 1225, 1242, respectively, using their mean value per basket calculated from 9 viz. 10 stations in the same depth range in the area, to account for different sizes. For Peltarion, this average value was 14, and for Eurypodius, it was 10. This procedure did not influence the data on species occurrence and distribution but enables us to arrive at rough data of species dominance. All other species were collected quantitatively from all hauls. Collected specimens were preserved aboard in $10 \%$ buffered formaldehyde.

Species identification was carried out using the following literature: Holthuis, 1952; Tiefenbacher, 1990 a, b (Caridea); Haig, 1955; Retamal,
1973,1992 (Anomura); Garth, 1957; Boschi et al., 1992 (Brachyura, Astacura, Palinura).

To compare the geographical distribution of the decapod fauna, we arbitrarily divided the area covered by the AGT hauls into three regions: (1) Strait of Magellan (52 $57^{\prime}$ 'S, $70^{\circ} 46^{\prime} \mathrm{W}-53^{\circ} 59^{\prime}$ 'S, $70^{\circ} 33^{\prime} \mathrm{W}$; 21 stations); (2) channel system between the Straits of Magellan and the Beagle Channel (54\%0'S, $71^{\circ} 00^{\prime} \mathrm{W}-54^{\circ} 58^{\prime} \mathrm{S}, 69^{\circ} 01^{\prime} \mathrm{W} ; 26$ stations), and (3) "southern islands" off the eastern mouth of the Bea-

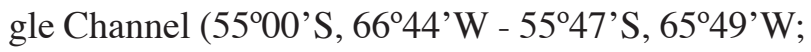
25 stations).

\section{RESULTS}

\section{Species composition and abundance}

The 72 AGT contained a total of 30 species (Table 1) comprising representatives of a total of 16 families (Caridea: 7 families/14 species; Astacura: 1 family/1 species; Palinura: 1 family/1 species; Anomura: 3 families/8 species; Brachyura: 4 families/6 species).

Hippolytidae (Caridea) with 5, and Lithodidae, Galatheidae (Anomura), and Majidae (Brachyura), each with 3 species, were the most important families in terms of species richness; all of the remaining families were represented by 1 or 2 species only. Several specimens of the genera Eualus and Metacrangon could not be identified to species level and may represent two species new to science (Holthuis, pers. comm.).

Of a total of $>30,000$ individuals caught during the two cruises, the overwhelming majority was contributed by Munida spp. due to its large share in the area south of the Beagle, where it contributed $>90 \%$ by number. However, in the Strait of Magellan this species had a numerical percentage of less than one-third, sharing its dominant position with the crabs Peltarion spinosulum and Eurypodius latreillii and the hermit crab Pagurus comptus (Fig. 1, above). In the channel system south of the Strait including the Beagle, Munida spp. was not among the dominants whereas $P$. spinosulum, $P$. comptus and E. latreillii continued to play an important part. In this area, also caridean shrimps were numerically dominant, above all Campylonotus semistriatus and Austropandalus grayi (Fig. 1, centre). South of the Beagle Channel most catches were entirely dominated by extremely high numbers of galatheids (Fig. 1 , bottom). 
TABLE 1. - Species list of decapods caught by AGT in the Magellan region ("Victor Hensen" and "Polarstern" material combined).

Straits of Magellan

Stat.-Nr Pa Pd Nl Nsp Cs Cv Bt Nm Ed Esp La Ct Ag M Tb Ss Lsa Pgr Psp Pc Pga MspMspp Mo Ela Elo LsmHp Ps Pv

\begin{tabular}{|c|c|c|c|c|c|c|c|c|c|c|c|c|c|c|c|c|c|c|c|c|c|c|c|c|c|c|c|c|c|}
\hline 805 & 0 & 0 & 0 & 0 & 0 & 0 & 0 & 0 & 0 & 0 & 0 & 0 & 0 & 0 & 0 & 0 & 0 & 0 & 0 & 0 & 0 & 0 & 1 & 0 & 0 & 0 & 0 & 0 & 18 \\
\hline VH 806 & 0 & 0 & 0 & 0 & 0 & 0 & 0 & 0 & 0 & 0 & 0 & 0 & 0 & 0 & 0 & 0 & 0 & 0 & 0 & 8 & 0 & 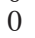 & 2 & 0 & 125 & 0 & 0 & 4 & 6 \\
\hline VH 812 & 0 & 0 & 0 & 0 & 0 & 0 & 0 & 0 & 0 & 0 & 0 & 0 & 0 & 0 & 0 & 0 & 0 & 0 & 0 & 25 & 0 & 0 & 1 & 0 & 107 & 0 & 0 & 1 & 10 \\
\hline VH 816 & 0 & 0 & 0 & 0 & 0 & 0 & 0 & 0 & 0 & 0 & 0 & 0 & 0 & 0 & 0 & 0 & 0 & 0 & 0 & 7 & 0 & 0 & 4 & 0 & 26 & 0 & 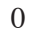 & 0 & 0 \\
\hline /H 821 & 0 & 0 & 0 & 0 & 0 & 0 & 0 & 0 & 0 & 0 & 0 & 0 & 0 & 0 & 0 & 0 & 0 & 0 & 0 & 13 & 0 & 0 & 84 & 0 & 5 & 0 & 0 & 0 & 20 \\
\hline VH 834 & 0 & 0 & 0 & 0 & 1 & 0 & 0 & 0 & 0 & 0 & 0 & 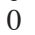 & 0 & 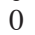 & 0 & 0 & 0 & 0 & 0 & 0 & 0 & 0 & 0 & 0 & 0 & 0 & 0 & 0 & 105 \\
\hline H 846 & 0 & 0 & 0 & 0 & 5 & 0 & 0 & 0 & 0 & 0 & 0 & 0 & 0 & 0 & 0 & 0 & 0 & 0 & 0 & 0 & 0 & 0 & 0 & & 0 & ) & & 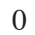 & 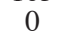 \\
\hline VH 861 & 0 & 0 & 0 & 0 & 0 & 0 & 0 & 0 & 0 & 0 & 0 & 0 & 1 & 0 & 0 & 0 & 0 & 0 & 0 & 11 & 0 & 0 & 31 & 0 & 30 & 3 & 0 & 0 & 176 \\
\hline Н 863 & 0 & 0 & 0 & 0 & 0 & 0 & 0 & 0 & 0 & 0 & 0 & 0 & 0 & 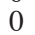 & 0 & 0 & 0 & 0 & 0 & 0 & 0 & 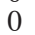 & 0 & 0 & 0 & 1 & 0 & 0 & 0 \\
\hline Н 865 & 1 & 0 & 0 & 0 & 2 & 0 & 0 & 0 & 0 & 0 & 0 & 0 & 0 & 0 & 0 & 0 & 0 & 0 & 0 & 0 & 0 & 0 & 0 & 0 & 0 & 0 & 0 & 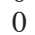 & 0 \\
\hline $\mathrm{H}$ & 0 & 0 & 0 & 0 & 0 & 0 & 0 & 0 & 0 & 0 & 0 & 0 & 0 & 0 & 0 & 0 & 0 & 0 & 0 & 0 & 0 & 0 & 0 & 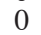 & 0 & 0 & 0 & 0 & 0 \\
\hline H 875 & 0 & 0 & 0 & 0 & 15 & 0 & 0 & 0 & 0 & 0 & 0 & 0 & 0 & 0 & 0 & 0 & 0 & 0 & 0 & 0 & 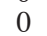 & 0 & 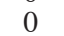 & & 0 & 0 & 0 & 0 & 0 \\
\hline VH 881 & 0 & 0 & 0 & 0 & 0 & 0 & 0 & 0 & 0 & 0 & 0 & 0 & 0 & 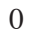 & 0 & 0 & 0 & 0 & 0 & 0 & 0 & 0 & 1 & 0 & 0 & 0 & 0 & 0 & 0 \\
\hline H 888 & 0 & 0 & 0 & 0 & 12 & 1 & 0 & 0 & 0 & 0 & 0 & 0 & 5 & U & 0 & 0 & 0 & 0 & 0 & 0 & 1 & 0 & 0 & & 5 & 0 & 0 & J & 4 \\
\hline H 920 & 0 & 0 & 0 & 0 & 0 & 0 & 0 & 0 & 0 & 0 & 0 & 0 & 0 & 0 & 0 & 0 & 0 & 0 & 0 & 5 & 0 & 0 & 585 & 1 & 19 & 0 & 0 & 0 & 41 \\
\hline VH 926 & 0 & 0 & 0 & 0 & 0 & 0 & 0 & 0 & 0 & 0 & 0 & 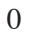 & 0 & 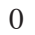 & 0 & 0 & 0 & 0 & 0 & 254 & 0 & 0 & 5 & & 63 & 0 & 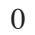 & 0 & 52 \\
\hline$H 04$ & 0 & 0 & 0 & 0 & 0 & 0 & 0 & 0 & 0 & 0 & 0 & 0 & 0 & U & 0 & 0 & 0 & 0 & 0 & 15 & 0 & 0 & 139 & ( & 32 & 4 & 0 & 0 & 386 \\
\hline H 95 & 0 & 0 & 0 & 0 & 0 & 0 & 0 & 0 & 0 & 0 & 0 & 0 & 0 & 0 & ( & 0 & 0 & 0 & 0 & 70 & 0 & 0 & 2 & & 46 & 0 & 0 & 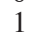 & 0 \\
\hline 960 & 0 & 0 & 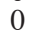 & 0 & 0 & 0 & 0 & 0 & 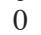 & 0 & & 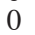 & 0 & & & 0 & 0 & & 0 & 69 & & & 18 & & 80 & & & & 12 \\
\hline VH 969 & 0 & 0 & 0 & 0 & 0 & 0 & 0 & 0 & 0 & 0 & 0 & 0 & 0 & 0 & 0 & 0 & 0 & 0 & 0 & 2 & 0 & 0 & 2 & & 9 & 1 & 0 & 0 & 14 \\
\hline VH 976 & 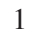 & 0 & 0 & 0 & 0 & 0 & 0 & 0 & 0 & 0 & 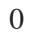 & . & 0 & 0 & 0 & 0 & 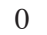 & 0 & 促 & 0 & 0 & 0 & 0 & 0 & 0 & 0 & 0 & 0 & 0 \\
\hline
\end{tabular}

Channel system south of Straits of Magellan

Stat.-Nr. Pa Pd Nl Nsp Cs Cv Bt Nm Ed Esp La Ct Ag M Tb Ss LsaPgrPgsp Pc Pga MspMspp Mo Ela Elo LsmHp Ps Pv

VH 1031

$\begin{array}{llllllllllllllllllllllllllllllllll}\text { VH } 1036 & 0 & 0 & 0 & 0 & 0 & 1 & 0 & 0 & 0 & 0 & 0 & 0 & 5 & 0 & 0 & 0 & 0 & 0 & 0 & 0 & 1 & 0 & 0 & 0 & 1 & 0 & 0 & 0 & 1 & 0\end{array}$

$\begin{array}{llllllllllllllllllllllllllllllll}\mathrm{VH} & 1042 & 0 & 0 & 0 & 0 & 16 & 0 & 0 & 0 & 0 & 0 & 0 & 0 & 0 & 0 & 0 & 0 & 0 & 0 & 0 & 0 & 0 & 0 & 0 & 0 & 0 & 0 & 0 & 0 & 0 & 0\end{array}$

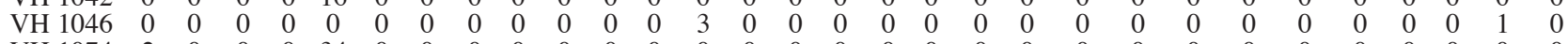

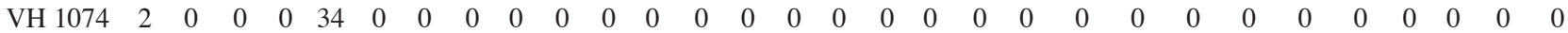

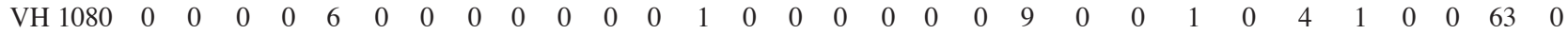

$\begin{array}{llllllllllllllllllllllllllllllll}\text { VH } 1086 & 0 & 0 & 0 & 0 & 2 & 0 & 0 & 0 & 0 & 0 & 0 & 0 & 0 & 0 & 0 & 0 & 0 & 0 & 0 & 0 & 0 & 0 & 0 & 0 & 0 & 0 & 0 & 0 & 0 & 0 \\ \text { VH } & 1107 & 0 & 0 & 0 & 0 & 0 & 0 & 0 & 0 & 0 & 0 & 0 & 0 & 0 & 0 & 0 & 0 & 1 & 0 & 0 & 0 & 0 & 0 & 0 & 0 & 0 & 0 & 0 & 0 & 1 & 0\end{array}$

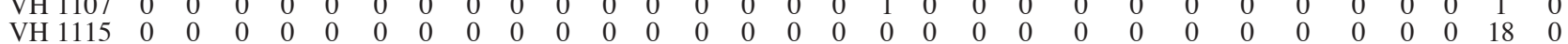

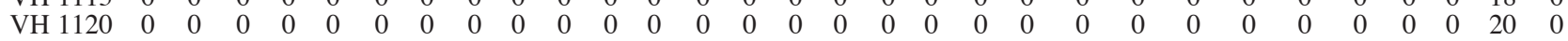

$\begin{array}{lllllllllllllllllllllllllllllll} & \mathrm{VH} \\ 1121 & 0 & 0 & 0 & 0 & 1 & 0 & 0 & 0 & 0 & 0 & 0 & 0 & 0 & 0 & 0 & 0 & 0 & 0 & 0 & 0 & 0 & 0 & 0 & 0 & 0 & 0 & 0 & 0 & 2 & 0\end{array}$

$\begin{array}{llllllllllllllllllllllllllllllll}\mathrm{VH} & 1133 & 6 & 1 & 0 & 0 & 1 & 0 & 0 & 0 & 0 & 0 & 0 & 0 & 0 & 0 & 0 & 0 & 0 & 0 & 0 & 0 & 0 & 0 & 0 & 0 & 0 & 0 & 0 & 0 & 1 & 0\end{array}$

$\begin{array}{llllllllllllllllllllllllllllllll}\mathrm{VH} & 1137 & 4 & 0 & 0 & 0 & 2 & 0 & 0 & 0 & 0 & 0 & 0 & 0 & 0 & 0 & 0 & 0 & 0 & 0 & 0 & 0 & 0 & 0 & 0 & 0 & 0 & 0 & 0 & 0 & 2 & 0\end{array}$

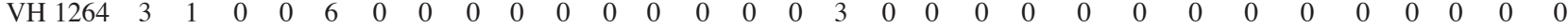

$\begin{array}{lllllllllllllllllllllllllllllllllllll}\mathrm{VH} & 1269 & 2 & 0 & 0 & 0 & 2 & 0 & 0 & 0 & 0 & 0 & 0 & 0 & 0 & 0 & 0 & 3 & 0 & 0 & 0 & 0 & 0 & 0 & 0 & 0 & 0 & 0 & 0 & 0 & 0 & 0 \\ \mathrm{VH} 1274 & 0 & 0 & 0 & 0 & 0 & 0 & 0 & 0 & 0 & 0 & 0 & 0 & 0 & 0 & 0 & 0 & 0 & 0 & 0 & 0 & 0 & 0 & 0 & 0 & 0 & 0 & 0 & 0 & 0 & 0\end{array}$

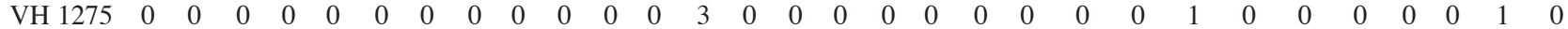

$\begin{array}{lllllllllllllllllllllllllllllllllll}\mathrm{VH} & 1277 & 3 & 1 & 0 & 0 & 34 & 0 & 0 & 0 & 0 & 0 & 0 & 0 & 0 & 0 & 0 & 0 & 0 & 0 & 0 & 0 & 0 & 0 & 0 & 0 & 0 & 0 & 0 & 0 & 0 & 0\end{array}$

VH 1278 0 $10 \begin{array}{llllllllllllllllllllllllllllll} & 0 & 0 & 0 & 0 & 0 & 0 & 0 & 0 & 0 & 0 & 0 & 0 & 0 & 0 & 0 & 0 & 0 & 0 & 0 & 0 & 0 & 0 & 0 & 0 & 0 & 0 & 0 & 0\end{array}$

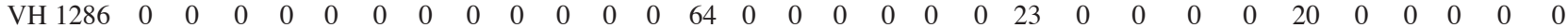

$\begin{array}{lllllllllllllllllllllllllllllll}\text { VH } 1290 & 0 & 0 & 0 & 0 & 0 & 0 & 0 & 0 & 0 & 0 & 0 & 0 & 0 & 0 & 0 & 0 & 0 & 0 & 0 & 0 & 0 & 0 & 1 & 0 & 9 & 0 & 9 & 0 & 6 & 0\end{array}$

$\begin{array}{lllllllllllllllllllllllllllllllllllll}\mathrm{VH} & 1294 & 0 & 0 & 0 & 0 & 0 & 0 & 0 & 0 & 0 & 0 & 0 & 0 & 0 & 0 & 0 & 0 & 0 & 0 & 0 & 0 & 0 & 0 & 0 & 0 & 0 & 0 & 0 & 0 & 0 & 0\end{array}$

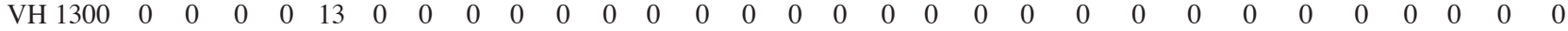

$\begin{array}{llllllllllllllllllllllllllllllll}\mathrm{VH} & 1306 & 0 & 0 & 0 & 0 & 4 & 0 & 0 & 0 & 0 & 0 & 0 & 0 & 0 & 0 & 0 & 0 & 0 & 0 & 0 & 0 & 0 & 0 & 0 & 0 & 0 & 0 & 1 & 0 & 21 & 0\end{array}$

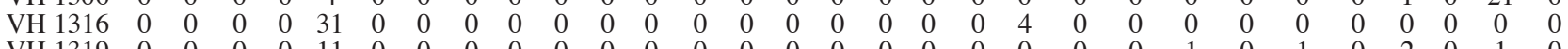

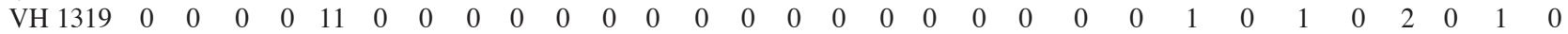

Southern islands

Stat.-Nr. Pa Pd Nl Nsp Cs Cv Bt Nm Ed Esp La Ct Ag M Tb Ss Lsa Pgr Psp Pe Pga MspMspp Mo Ela Elo LsmHp Ps Pv

$\begin{array}{llllllllllllllllllllllllllllllll}\mathrm{VH} & 1141 & 0 & 0 & 0 & 0 & 4 & 0 & 0 & 0 & 0 & 0 & 0 & 0 & 0 & 0 & 0 & 0 & 2 & 0 & 0 & 0 & 0 & 0 & 20 & 0 & 0 & 0 & 0 & 0 & 10 & 0\end{array}$ $\begin{array}{llllllllllllllllllllllllllllllllllll}\text { VH } 1149 & 0 & 0 & 0 & 0 & 0 & 3 & 0 & 3 & 0 & 0 & 0 & 0 & 74 & 0 & 0 & 0 & 0 & 0 & 0 & 39 & 0 & 0 & 2945 & 0 & 75 & 3 & 0 & 4 & 60 & 1\end{array}$ $\begin{array}{llllllllllllllllllllllllllllllll}\mathrm{VH} & 1153 & 0 & 0 & 0 & 0 & 0 & 0 & 4 & 33 & 1 & 0 & 0 & 0 & 1 & 0 & 0 & 0 & 0 & 2 & 0 & 1 & 0 & 0 & 432 & 0 & 16 & 0 & 0 & 0 & 71 & 0\end{array}$

$\begin{array}{llllllllllllllllllllllllllllllllll}\mathrm{VH} & 1158 & 0 & 0 & 0 & 0 & 0 & 1 & 1 & 0 & 0 & 0 & 0 & 0 & 23 & 0 & 0 & 0 & 1 & 0 & 0 & 1 & 0 & 0 & 423 & 0 & 40 & 2 & 0 & 0 & 52 & 0\end{array}$

VH 1162 0 $10 \begin{array}{llllllllllllllllllllllllllllllllllll} & 0 & 0 & 0 & 0 & 0 & 0 & 0 & 0 & 0 & 0 & 0 & 0 & 0 & 0 & 3 & 3 & 0 & 81 & 0 & 0 & 223 & 0 & 11 & 0 & 0 & 0 & 59 & 0\end{array}$

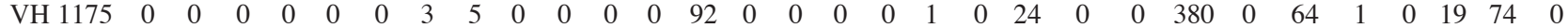

$\begin{array}{llllllllllllllllllllllllllllllllll}\mathrm{VH} & 1182 & 0 & 0 & 0 & 0 & 0 & 0 & 0 & 0 & 0 & 0 & 0 & 0 & 2 & 0 & 0 & 0 & 0 & 1 & 0 & 1 & 0 & 0 & 115 & 0 & 1 & 0 & 0 & 0 & 0 & 0\end{array}$

$\begin{array}{lllllllllllllllllllllllllllllllllll}\text { VH } 1191 & 0 & 0 & 0 & 0 & 0 & 6 & 1 & 4 & 0 & 0 & 0 & 0 & 159 & 0 & 0 & 0 & 0 & 3 & 0 & 23 & 0 & 0 & 936 & 0 & 8 & 0 & 0 & 0 & 49 & 2\end{array}$

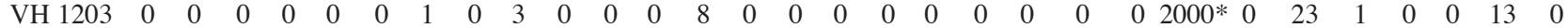

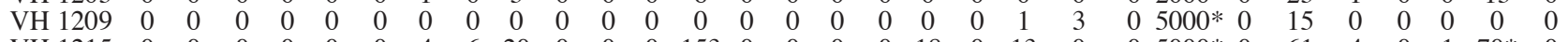

$\begin{array}{llllllllllllllllllllllllllllllllllllll}\mathrm{VH} & 1215 & 0 & 0 & 0 & 0 & 0 & 0 & 4 & 6 & 20 & 0 & 0 & 0 & 153 & 0 & 0 & 0 & 0 & 18 & 0 & 13 & 0 & 0 & 5000 * & 0 & 61 & 4 & 0 & 1 & 70 * & 0\end{array}$

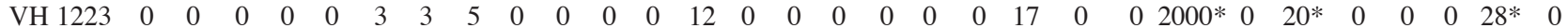

$\begin{array}{llllllllllllllllllllllllllllllllll}\text { VH } 1225 & 0 & 0 & 0 & 0 & 0 & 0 & 0 & 0 & 0 & 0 & 0 & 0 & 0 & 0 & 0 & 0 & 0 & 0 & 0 & 0 & 0 & 0 & 1000 * & 0 & 10 * & 0 & 0 & 0 & 14 * & 0\end{array}$

$\begin{array}{lllllllllllllllllllllllllllllllll}\text { VH } 1228 & 0 & 0 & 0 & 0 & 0 & 2 & 0 & 1 & 0 & 0 & 0 & 0 & 23 & 0 & 0 & 0 & 0 & 0 & 0 & 71 & 0 & 0 & 584 & 0 & 40 & 3 & 0 & 0 & 26 & 0\end{array}$

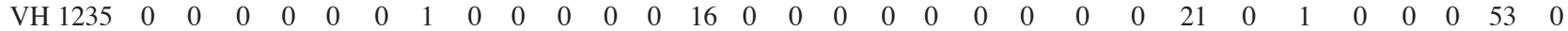


TABLE 1. (Cont.) - Species list of decapods caught by AGT in the Magellan region ("Victor Hensen" and "Polarstern" material combined).

\section{Southern islands}

Stat.-Nr Pa Pd Nl Nsp Cs Cv Bt Nm Ed Esp La Ct Ag M Tb Ss Lsa Pgr Psp Pc Pga MspMspp Mo Ela Elo LsmHp Ps Pv

\begin{tabular}{lccccccccccccccccccccccccccccccccccccccccc}
\hline VH 1242 & 0 & 0 & 0 & 0 & 0 & 0 & 0 & 0 & 0 & 0 & 0 & 0 & 13 & 0 & 0 & 0 & 0 & 0 & 0 & 36 & 0 & 0 & $3500 *$ & 0 & $35 *$ & 0 & 0 & 0 & $49 *$ & 0 \\
PS 106 & 0 & 0 & 1 & 0 & 0 & 0 & 0 & 0 & 0 & 0 & 0 & 0 & 0 & 0 & 0 & 0 & 0 & 0 & 0 & 0 & 0 & 0 & 0 & 0 & 0 & 0 & 0 & 0 & 0 & 0 \\
PS 107 & 0 & 0 & 28 & 0 & 0 & 0 & 0 & 0 & 0 & 0 & 0 & 0 & 0 & 0 & 0 & 0 & 0 & 0 & 0 & 0 & 0 & 0 & 0 & 0 & 5 & 0 & 0 & 0 & 0 & 0 \\
PS 108 & 0 & 0 & 0 & 0 & 0 & 0 & 0 & 0 & 0 & 0 & 0 & 0 & 0 & 0 & 0 & 0 & 0 & 0 & 0 & 2 & 0 & 0 & 0 & 0 & 0 & 0 & 0 & 0 & 0 & 0 \\
PS 109 & 0 & 0 & 0 & 0 & 3 & 0 & 0 & 1 & 0 & 2 & 0 & 0 & 1 & 4 & 0 & 1 & 0 & 0 & 1 & 23 & 0 & 0 & 19 & 0 & 0 & 0 & 0 & 0 & 0 & 0 \\
PS 110 & 0 & 0 & 0 & 0 & 1 & 3 & 1 & 12 & 17 & 0 & 0 & 0 & 131 & 0 & 0 & 0 & 0 & 2 & 0 & 18 & 0 & 0 & 1 & 0 & 32 & 0 & 0 & 0 & 0 & 0 \\
PS 111 & 0 & 0 & 72 & 1 & 9 & 0 & 0 & 0 & 0 & 0 & 0 & 0 & 0 & 0 & 0 & 1 & 0 & 0 & 0 & 0 & 0 & 0 & 11 & 0 & 0 & 0 & 0 & 0 & 1 & 0 \\
PS 114 & 0 & 0 & 6 & 0 & 1 & 0 & 0 & 0 & 0 & 0 & 1 & 0 & 0 & 0 & 1 & 0 & 0 & 0 & 0 & 0 & 0 & 0 & 0 & 0 & 0 & 0 & 0 & 0 & 0 & 0 \\
PS 115 & 1 & 0 & 0 & 0 & 1 & 0 & 0 & 1 & 0 & 1 & 0 & 1 & 0 & 11 & 0 & 0 & 0 & 0 & 0 & 2 & 0 & 0 & 47 & 0 & 0 & 0 & 0 & 0 & 0 & 0 \\
PS 117 & 0 & 0 & 0 & 0 & 0 & 0 & 0 & 2 & 0 & 0 & 0 & 0 & 0 & 0 & 0 & 0 & 0 & 0 & 0 & 0 & 0 & 4 & 0 & 0 & 0 & 0 & 0 & 0 & 2 & 0
\end{tabular}

Abbreviations (in alphabetical order). Ag: Austropandalus grayi; Bt: Betaeus truncatus; Cs: Campylonotus semistriatus; Ct: Chorismus tuberculatus; Cv: Campylonotus vagans; Ed: Eualus dozei; Ela: Eurypodius latreillii; Elo: Eurypodius longirostris; Esp: Eualus sp.; Hp: Halicarcinus planatus; La: Lebbeus antarcticus; Lsm: Libidoclaea smithii; Lsa: Lithodes santolla; M: Metacrangon sp.; Mo: Munidopsis opalescens; Mspp: Munida spp. (M. subrugosa and M. gregaria); Msp: Munida spinosa; Nl: Nematocarcinus lanceopes; Nm: Nauticaris magellanica; Nsp: Nematocarcinus sp.; Pa; Pasiphaea acutifrons; Pc: Pagurus comptus; Pd: Pasiphaea dofleini; Pga: Pagurus gaudichaudii; Pgr: Paralomis granulosa; Ps: Peltarion spinosulum; Psp: Paralomis spinosissima; Pv: Pinnixia valdiviensis; Ss: Stereomastis suhmi; Tb: Thymops birsteini

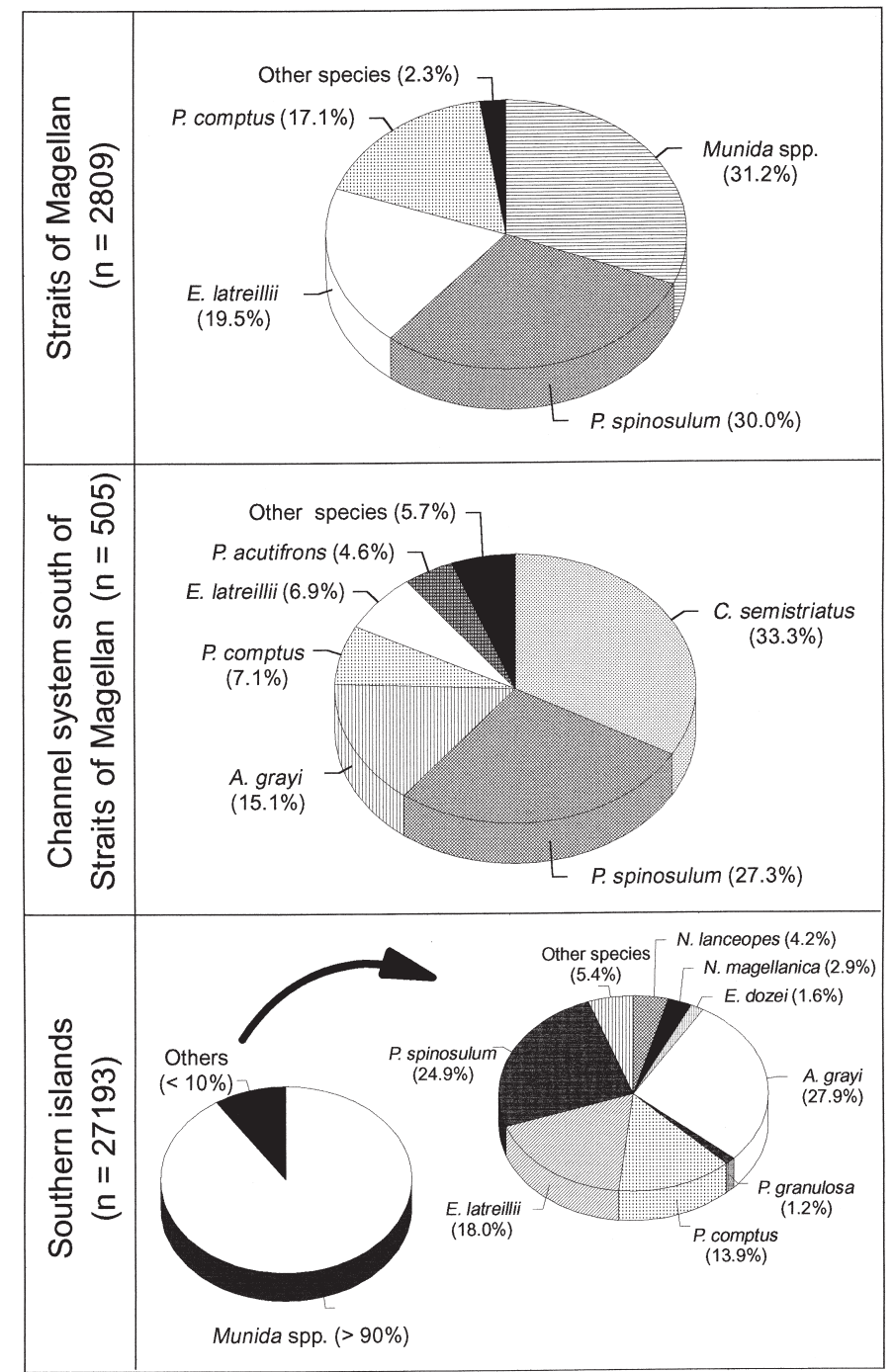

FIG. 1. - Shares of dominant decapod species in AGT samples obtained from the areas of investigation ("Victor Hensen" and "Polarstern" cruises combined). Total number of decapods caught in each area is indicated on the left. Numbers of 3 principal dominants from the "southern islands" area are estimates, see Material and Methods 

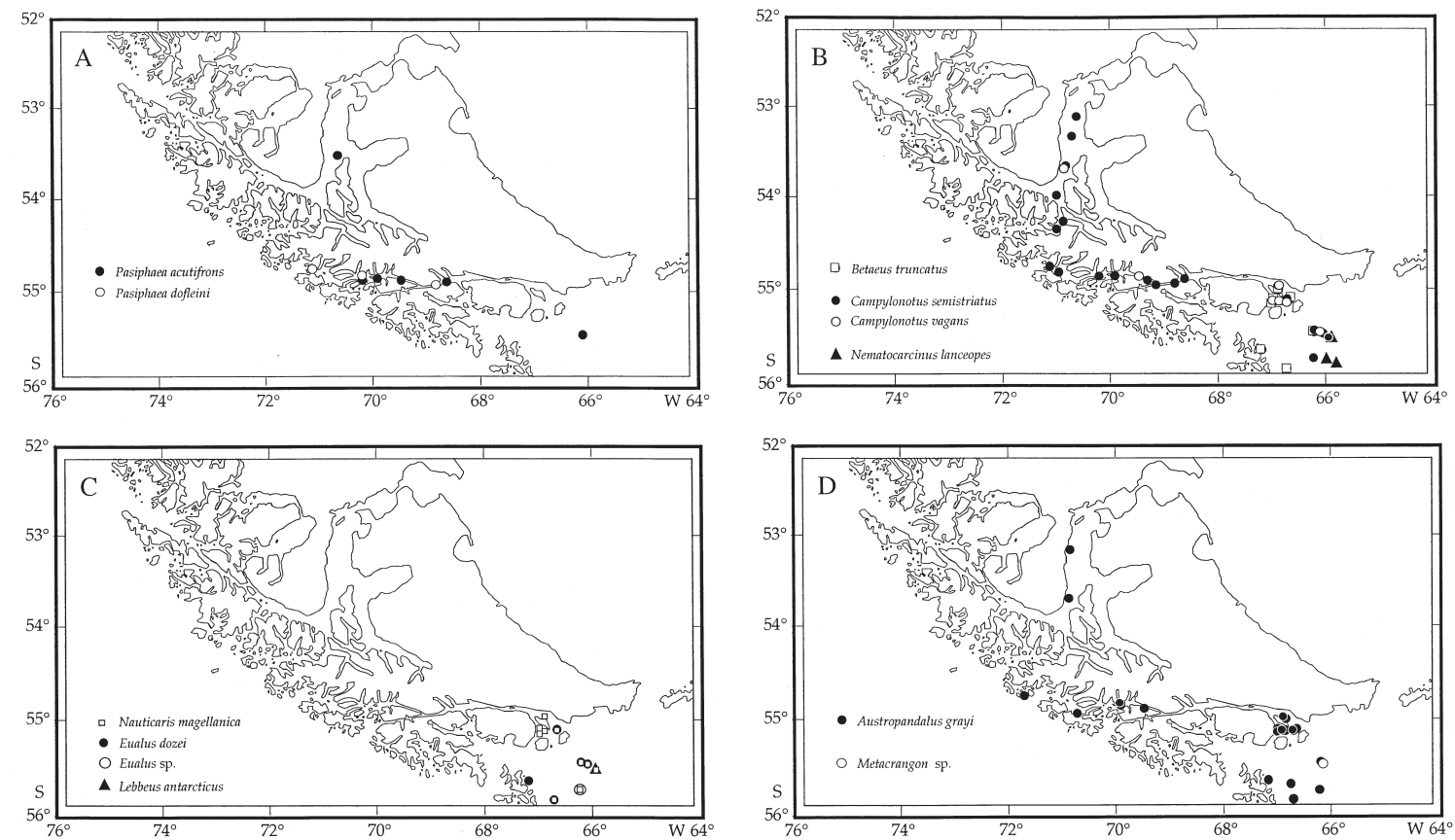

FIG. 2. - Locations for caridean shrimps collected during the "Victor Hensen" campaign, 1994: A Pasiphaeidae; B AlpheidaeCampylonotidae-Nematocarcinidae; C Hippolytidae; D Pandalidae - Crangonidae.
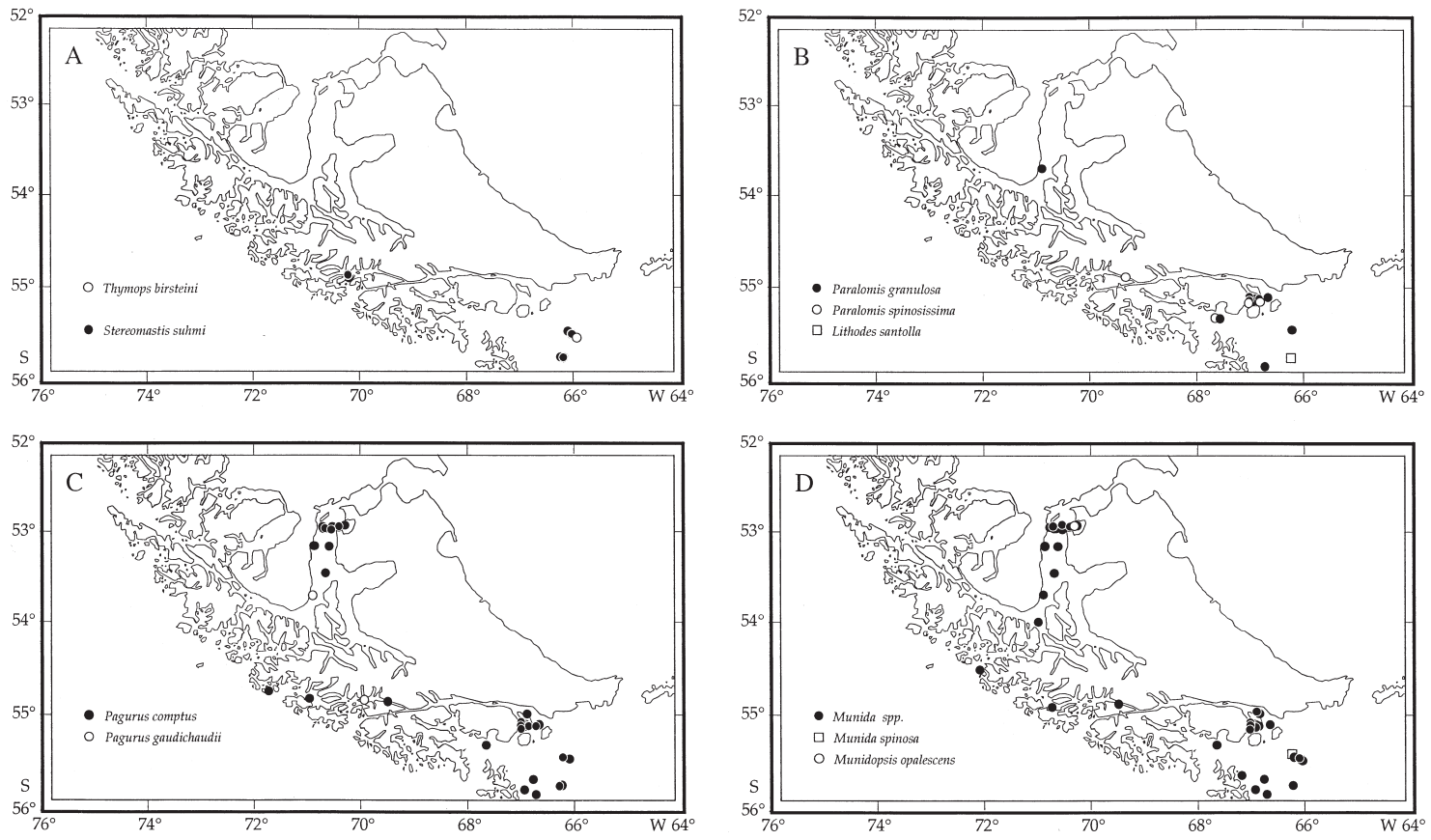

FIG. 3. - Locations for astacuran and palinuran lobsters and anomuran crabs collected during the "Victor Hensen" campaign, 1994: A Astacura - Palinura; B Lithodidae; C Paguridae; D Galatheidae.

\section{Regional distribution}

Decapod crustaceans were common within all three areas of investigation, however, species number varied between the regions. The regional distribution of selected species is shown in Figures 2-4. South of the Beagle Channel, a total of 27 species occurred; only Pasiphaea dofleini, Munidopsis opalescens and Libidoclaea smithii were missing in this area. A total of 14 species was found in the Strait of Magellan and 13 species were distributed in the channels located between the Strait and the eastern mouth of the Beagle Channel. 

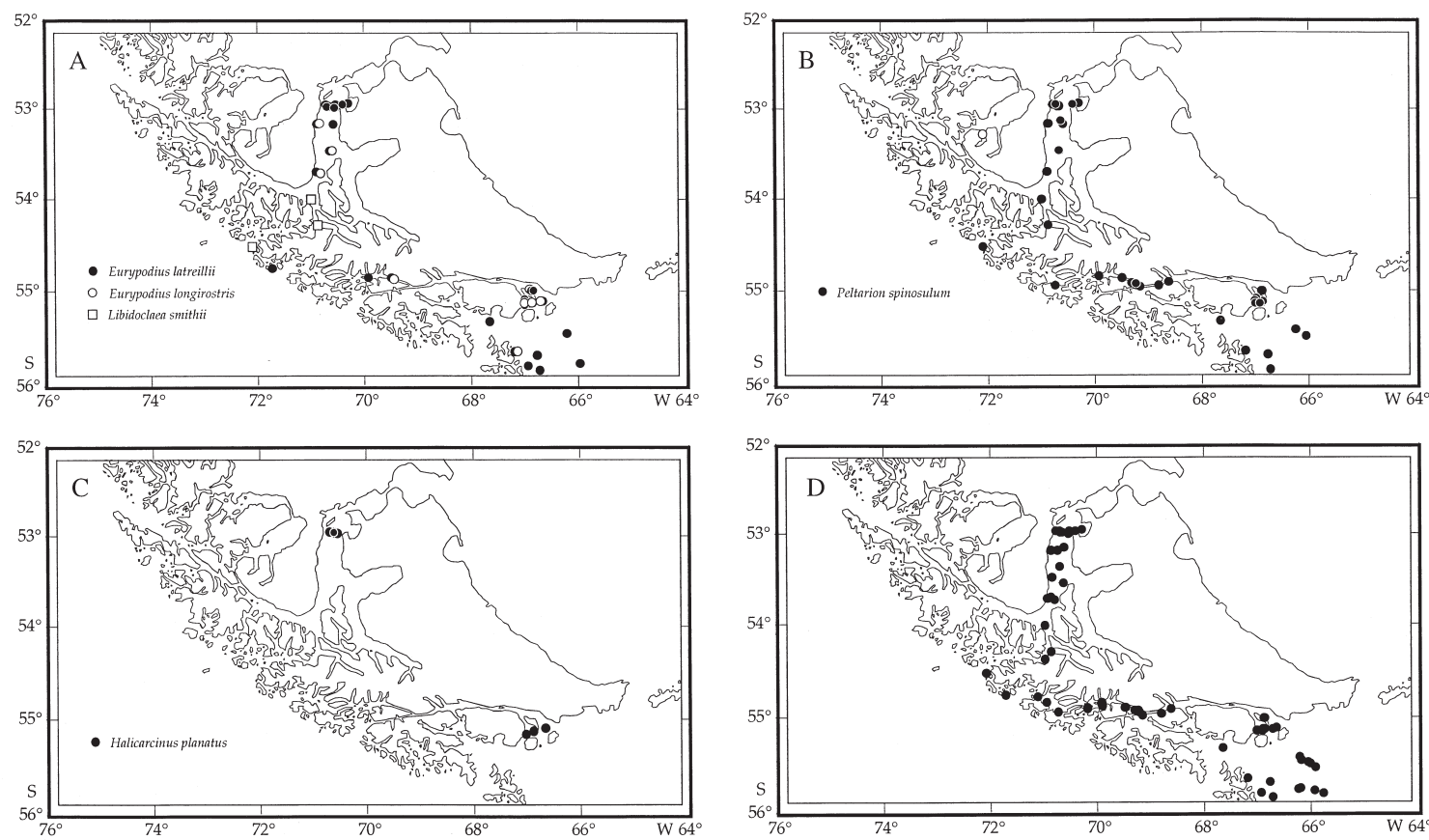

FIG. 4. - Locations for brachyuran crabs collected during the "Victor Hensen" campaign, 1994: A Majidae; B Atelecyclidae; C Hymenosomatidae; D Location of sampling sites ("Victor Hensen"-cruise and "Polarstern"cruise ANT XIII/4 combined).

Nine species were found in all three areas, whereas all other decapod crustaceans revealed distinct horizontal distribution ranges (cf. Table 1). The widest geographic distribution was demonstrated by the two brachyuran crabs $P$. spinosulum and $E$. latreillii, which were present between the northwesternmost station in the Straits of Magellan and the southernmost point of investigation, on the continental slope south of the Beagle Channel. The most restricted range was found in all members of the Hippolytidae (Caridea), which occurred only south of the Beagle Channel. Rare species such as Metacrangon sp., Eualus spp., Lebbeus antarcticus, Nematocarcinus lanceopes and Thymops birsteini have been recorded only far in the south, and Stereomastis suhmi has been found exclusively in the channel system.

Species with the greatest frequency of occurrence were $P$. spinosulum (Brachyura: Atelecyclidae), Munida spp. (Anomura: Galatheidae), present in 38 and 35 hauls, respectively, E. latreillii (Brachyura: Majidae), present in 32 and $P$. comptus (Anomura: Paguridae), present in 30 hauls. Also the caridean shrimps $C$. semistriatus (Campylonotidae) and $A$. grayi (Pandalidae) were well represented with 26 and 21 records, respectively. All the remaining species were less common (less than $10 \%$ occurrence).

\section{Bathymetric distribution}

The median depth where species have been collected is shown in Figure 5, revealing also the respective minima and maxima. The largest species group $(\mathrm{n}=11)$ occurred mainly between 50 and $100 \mathrm{~m}$ depth; 7 of them (3 Caridea, 3 Anomura and 1 Brachyura) were restricted to the range above $110 \mathrm{~m}$. Exceptions within this shallow-water group were Nauticaris magellanica, P. comptus, Munida spp., E. latreillii and $P$. spinosulum, which revealed a much wider depth range. A second group, represented by 6 species, was obtained from depths ranging between approximately 300 and $800 \mathrm{~m}$. Representatives of this medium-water group generally showed a wider bathymetric distribution, as can be seen from the extremes in Figure 5. A depth range between $214 \mathrm{~m}$ and $480 \mathrm{~m}$ places Libidoclaea smithii in between the first two groups. Four species (Nematocarcinus lanceopes, Nematocarcinus sp., Lebbeus antarcticus and Thymops birsteini) were recorded exclusively from stations well below $1000 \mathrm{~m}$.

The species with the widest vertical distribution ranges were $C$. semistriatus, from 70-2165 m, $E$. latreillii, found between 9 and $1507 \mathrm{~m}$, and P. spinosulum occurring between 9 and $1270 \mathrm{~m}$. $N$. lanceopes was caught between 1270 and $2505 \mathrm{~m}$ depth. 


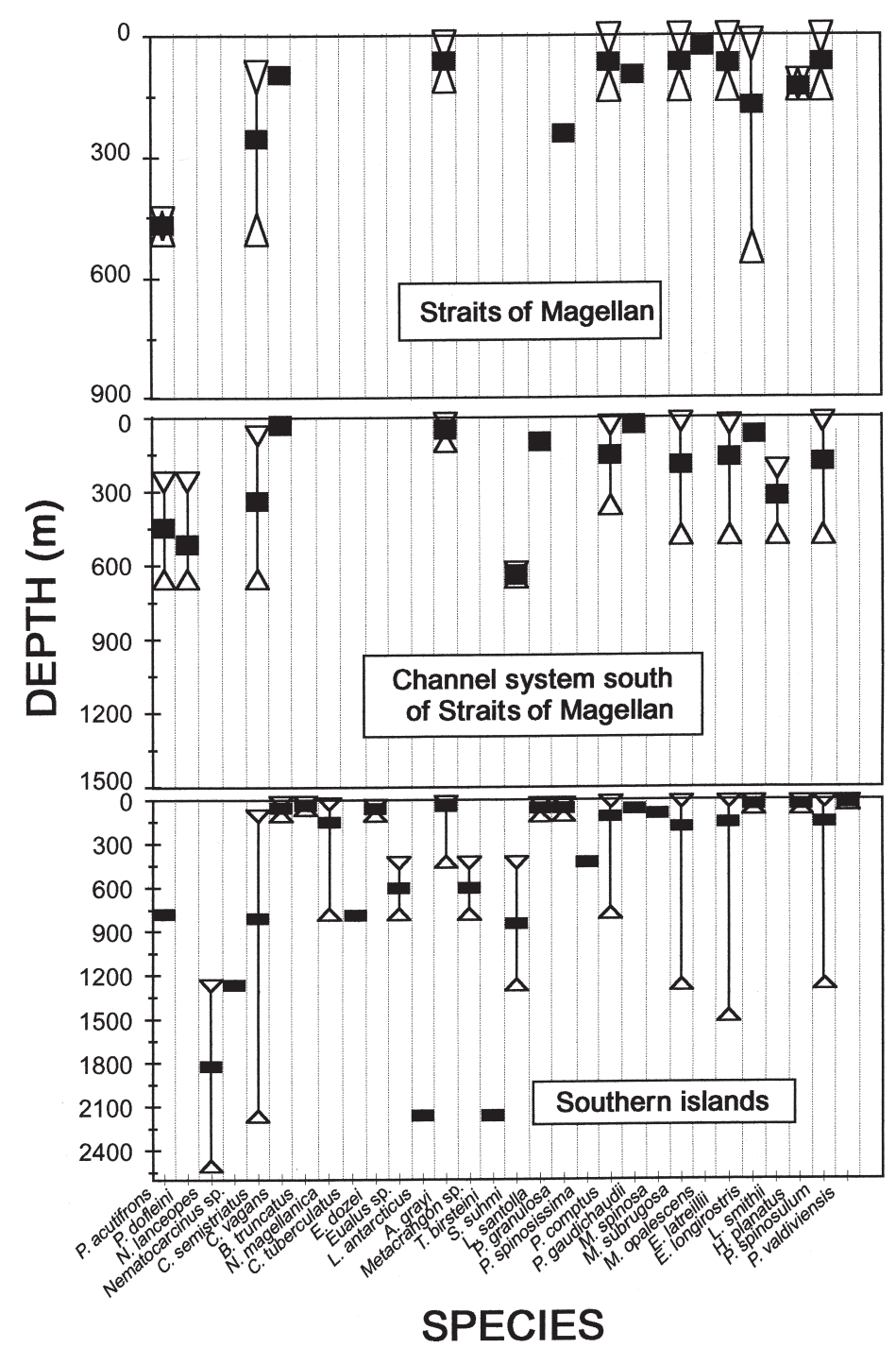

FIG. 5. - Depth ranges (medians and extremes) of decapod species in the three study areas.

\section{DISCUSSION}

The type of gear used in this study, which provided the most substantial material ever obtained in the Magellan region, does not yield strictly quantitative samples (see Ekau, 1988, and Voss, 1988), but it provides large amounts of material with relatively little effort and can be used for large-scale comparisons of benthic epifauna. For this reason numbers and percentages of the different species caught should be considered crude estimates, even more so as some numbers had to be recalculated (see Methods). As an additional means for estimating abundance, we took photographs of each haul and noted the biomass of the catch. Anyway, we can use this information only to compare benthic species. Only two of the 13 pelagic shrimp species known for the South American antiboreal zone have been caught whereas all known benthic caridean shrimp species were taken. For this reason, pelagic decapods will not be discussed further.

More than half of the 50 species, which are distributed between the Pacific entrance of the Straits of Magellan and Cape Horn (Gorny, 1999) have been caught on both expeditions during 1994 and 1996, among them two species likely to be new to science (Table 2). Furthermore, the known distribution range of 12 species was extended towards the south. Most benthic species formerly recorded but missing in our samples are anomuran and brachyuran crab species distributed between 0 and $250 \mathrm{~m}$ depth. As $65 \%$ of our AGT samples were carried out in this depth zone, the relatively poor presence of crab species in the samples is at first glance surprising. However, no catches were taken from the intertidal and shallow subtidal $<9 \mathrm{~m}$ which might have provided further species, e.g., the 
brachyurans Halicarcinus planatus and Acanthocyclus albatrossis. On the other hand, some of the species encountered had very high densities. Various catches close to the Atlantic entrance of the Straits of Magellan and south of the Beagle Channel yielded several $\mathrm{kg}$ of $P$. spinosulum and even higher weights of Munida spp., the two dominant decapod species of the area, which occur on sandy bottoms and muddy grounds, respectively. Underwater camera transects (Gutt and Schickan, 1996) confirmed exceptionally high densities of anomuran and brachyuran decapods in these areas, which are in sharp contrast to video and camera transects from the high Antarctic (Gutt et al., 1991). From UW photos, a maximum density of 2560 specimens per $100 \mathrm{~m}^{2}$ of Munida spp. was calculated for stn. 1219 south of the Beagle, and average densities of 700 specimens per $100 \mathrm{~m}^{2}$ were encountered in the "crustacean community" both in the Paso Ancho area of the Straits of Magellan and south of the Beagle (Gutt, pers. comm.).

TABLE 2. - Species composition and distribution of decapods in the waters off Patagonia and Tierra del Fuego (this study) compared to earlier records in the Chilean part of the antiboreal region of South America and around Antarctica (* cf. Gorny, 1999).

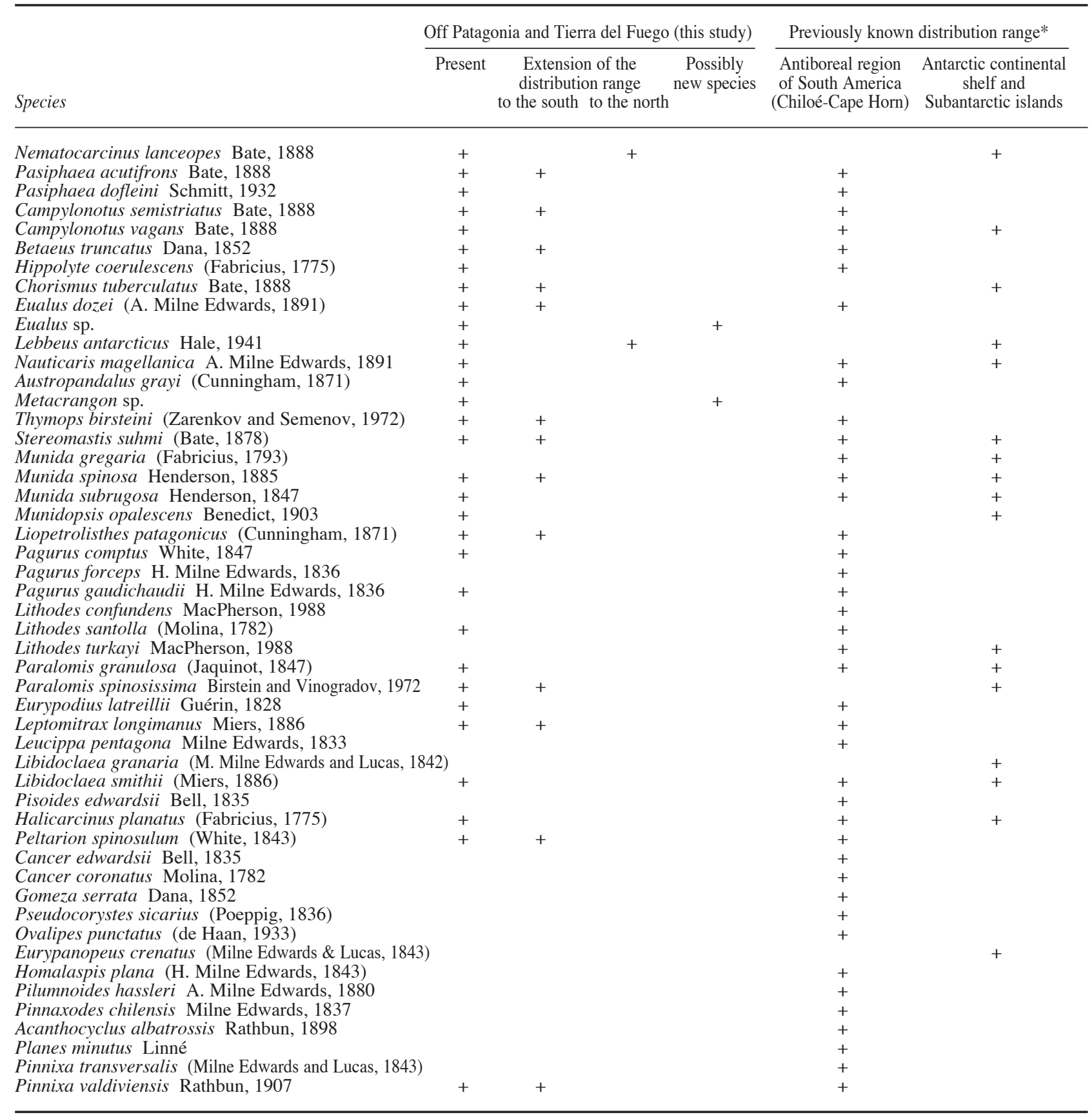


Depth ranges of AGT hauls differed substantially in the three areas of study during the two cruises. These ranges had to be accepted for technical reasons: "Victor Hensen" could not trawl much beyond $600 \mathrm{~m}$ due to the length of its wires, "Polarstern" found few trawlable shallow grounds on the northern slope of the Drake Passage, and both ships did not enter the shallow sublittoral. The poor species number from the hauls taken in the Straits of Magellan may also be a result of trawling depths. Some decapods which exhibit a high degree of eurybathy (E. latreillii, P. spinosulum, Munida spp.) were caught in large numbers in this area whereas other species were missing, among them typical shallowwater species such as the brachyuran A. albatrossis.

Despite this bias, formerly published depth ranges for some decapod species of the Magellan region (Holthuis, 1952; Zarenkov, 1970; Retamal, 1973,1974; Vinuesa, 1977) have been extended towards greater depths from our AGT catches for the following species: C. semistriatus, N. magellanica, L. antarcticus, A. grayi, Munida spp., P. comptus, E. latreillii, P. spinosulum, L. smithii and T. birsteini. On the other hand, some species were confirmed to be restricted to quite shallow waters hardly exceeding $100 \mathrm{~m}$ : B. truncatus, E. dozei, $C$. vagans, L. santolla, $P$. gaudichaudii, $H$. planatus and $P$. valdiviensis.

From the antiboreal zone towards the "true" Antarctic (i.e., south of the Antarctic Convergence) there is a steep decline in species richness of decapods (Gorny, 1999, with numerous references). Only 11 benthic and 12 pelagic decapod species have been recorded in these Antarctic waters which are characterized not only by very low temperatures but also an extremely short productive season, and 6 species are known from the continental slope and the deep-sea basins surrounding Antarctica. Towards the Antarctic continental shelf the species number of decapods is reduced once again; only 5 benthic and 3 pelagic species, all of them caridean shrimps, were reported by Arntz and Gorny (1991) from the Weddell sea shelf. These shrimps locally attain relatively high densities; from UW photographs, $>33$ Chorismus antarcticus per $100 \mathrm{~m}^{2}$ were calculated nearshore at Halley Bay, and of Nematocarcinus lanceopes up to 9 specimens per $100 \mathrm{~m}^{2}$ were counted in the deep Weddell Sea (Gutt et al., 1991). However, these values are 1-2 orders of magnitude lower than the anomuran densities cited above for the Magellan region. The only other decapod group reaching strictly high Antarctic waters are lithodid crabs (Anomura), with 2 species (Klages et al., 1995; Gorny, 1999). Brachyuran crabs, palinurans and astacurans are absent on the Antarctic continental shelves which may be due to the present-day existence of deep-sea areas between the Antarctic and the surrounding continents, but may have physiological causes as well (Frederich et al., in press). The large areas between the antiboreal zone and the Antarctic Convergence, on the other hand, reveal quite a high species richness of decapods as can be seen from Gorny's (1999) compilation. Particularly from the brachyurans and galatheid anomurans which are so common in the Magellan region and absent in the high Antarctic, one would expect considerable pressure to re-extend their area towards the Antarctic continent if the present warming trend continues (Arntz, 1998). However, contrary to the situation in the early Tertiary when decapod groups were still diverse and abundant around Antarctica (Zinsmeister and Feldmann, 1984; Feldmann and Tshudy, 1989; Crame, 1994), a shallow-water connection to South America is no longer existent.

This first detailed inventory of the Magellan decapod fauna after many years has largely clarified its biogeographic position and its relations to the Antarctic, where more intense sampling has been carried out during past years. The Magellan region as a refuge area in glacial times has maintained a much higher number of species and a greater diversity of decapod groups compared with the Antarctic proper which suffered extinction of many groups that seem to have flourished at least until the Eocene (Crame,1994). Our results reveal an overlap between species occurring in the Antarctic and the southern tip of the Magellan region which caused Gorny (1999) to subdivide the formerly described South American antiboreal fauna into a cold-temperate and a cold-water/Antarctic fauna. Lebbeus antarcticus, Nematocarcinus lanceopes, Stereomastis suhmi and Paralomis spinosissima were found for the first time south of the Beagle, i.e. on the northern slope of the Drake Passage. Additionally, two species probably new to science have been caught. $N$. lanceopes, which belongs to the deep-sea fauna of the Southern Ocean, was registered for the first time north of the Antarctic Convergence, as was L. antarcticus, a shrimp formerly known from the continental shelves of East and West Antarctica (Arntz and Gorny, 1991; Gorny, 1999). The specimens of $S$. suhmi were the southernmost adults ever caught, since only some larvae had appeared before in plankton samples taken close to South Georgia 
(Tiefenbacher, 1994). The other deep-sea species, the astacuran lobster T. birsteini, was known from the Atlantic sector before (cf. Boschi et al., 1981).

Thus, for the decapods, the southern tip of the Magellan region (or northern slope of the Drake Passage) does indeed seem to be a transitional area between the Antarctic and cold-temperate faunas. Our data appear to indicate species richness to be higher around the "southern islands" than in the Straits of Magellan and the channel system, but this may be a consequence of our sampling scheme (see above). Further sampling will have to reveal whether the formerly described pattern of decreasing species number with increasing latitude between Chiloé Island and Cape Horn continues to be valid (Gorny, 1999). Species numbers of most groups clearly decrease further into Antarctic waters. From our data it is obvious, however, that towards the Antarctic continent there is a second pattern: a shift from reptant to natant (caridean) decapods. Surprisingly some groups - above all the brachyurans and the galatheid anomurans - thrive well right up to the northern slope of the Drake Passage, where they even attain exceptionally high densities. The only group that has successfully reconquered the high Antarctic is the caridean shrimps whose high frequencies of occurrence and, sometimes, high densities are in contrast with their low number of species. Conversely, shrimps are rich in species but mostly reveal low densities in the Magellan area.

Future work should complete the inventory of the Magellan region (which is presently done on several "Vidal Gormaz" cruises; see Mutschke and Gorny, 1999) and intensify investigations on the southern slope of the Drake Passage using the same gear and methodology as in the Magellan region and the high Antarctic. This would be helpful in finding out to what extent the Drake Passage as a whole is a transitional area between the Antarctic Peninsula and Magellan waters. For this same purpose, cooperation should be intensified with Spanish scientists who have sampled the Scotia Arch for some years (see Ramos, 1999).

Furthermore, the rich decapod material obtained during the various cruises in the Magellan region should be used to extend the study of latitudinal gradients in population dynamics and reproductive biology encountered from the high to the low Antarctic (Gorny et al., 1992) into cold temperate waters. In this context it will be helpful that several Antarctic species also occur in the Magellan region, thus enabling a comparison on species level. Latitu- dinal gradients are under investigation also in decapod physiology (Pörtner, pers. comm.), aiming at clarifying potential physiological constraints that may cause the striking absence of some decapod groups from Antarctic waters.

\section{ACKNOWLEDGEMENT}

The authors would like to thank Dr. L.B. Holthuis who advised as to the proper spelling of some decapod names.

\section{REFERENCES}

Arntz, W.E. - 1998. Marine ecology in Antarctica and its connections to "global change". In: M. Picazzo (ed.), Atti del 12. Congresso dell'Associazione Italiana di Oceanologia e Limnologia, Vol. II, pp. 3-27. A.I.O.L., Genova.

Arntz W.E. and M Gorny. - 1991. Shrimp (Decapoda, Natantia) occurrence and distribution in the eastern Weddell Sea, Antarctica. Polar Biol., 11: 169-177.

Arntz, W. and M. Gorny. - 1996. Cruise report of the Joint ChileanGerman-Italian Magellan "Victor Hensen" Campaign in 1994. Ber. Polarforsch., 190, 1-113.

Boschi, E.E., M.I. Iorio and K. Fischbach. - 1981. Distribución y abundancia de los crustáceos decápodos capturados en las campañas de los B/I "Shinkai Maru" en el Mar Argentino, 1978-79. In: V. Angelescu (ed.), Campañas de investigación pesquera realizadas en el mar argentino por los B/I "Shinkai Maru" y "Walter Herwig" y el B/P "Marburg”, años 1978 y 1979. Resultados de la parte argentina, pp. 233-250.

Boschi, E.E., C.E. Fischbach and M.I. Iorio. - 1992. Catálogo ilustrado de los crustáceos estomatópodos y decápodos marinos de Argentina. Frente Marítimo, Montevideo, 10: 7-94.

Brattström, H. and E. Dahl. - 1951. Reports of the Lund Expedition 1948-49. 1. General account, list of stations, hydrography. Lunds Univ. Arsskr., N.F.2, 46 (8): 1-86.

Brattström, H., and A. Johanssen. - 1983. Ecological and regional zoogeography of the marine benthic fauna of Chile. Sarsia, 68: 289-339.

Crame, A.J. - 1994. Evolutionary history of Antarctica. In: G. Hempel (ed.), Antarctic Science - Global Concerns, pp. 188214. Springer Verlag, Berlin:

Doflein, F. and H. Balss. - 1912. Die Dekapoden und Stomatopoden der Hamburger Magalhaenischen Sammelreise 1892/93. Mittl. Naturhist. Mus., 24: 24-44.

Ekau, W. - 1988. Ökomorphologie notothenioider Fische aus dem Weddellmeer, Antarktis. Ber. Polarforsch., 51: 1-140.

Fahrbach, E. and D. Gerdes. - 1997. The expedition ANTARKTIS XIII/4-5 of the research vessel "Polarstern" in 1996. Ber. Polarforsch., 239: 1-126.

Feldmann, R.M. and P.G. Quilty. - 1997. First pliocene decapod crustacean (Malacostraca: Palinuridae) from the Antarctic. Antarct. Sci., 9: 56-60.

Feldmann, R.M. and D.M. Tshudy. - 1989. Evolutionary patterns in macrurous decapod crustaceans from Cretaceous to early Cenozoic rocks of the James Ross Island region, Antarctica. In: J. A. Crame (ed.), Origins and Evolution of the Antarctic Biota. Geol. Soc. Spec. Publ., 47: 183-195.

Frederich, M., F.J. Sartorius, W.E. Arntz and H.-O. Pörtner. - in press. Haemolymph magnesium regulation in decapod crustaceans: physiological correlates and ecological consequences in polar areas. J. Exp. Biol.

Garth, J.S. - 1957. Reports of the Lund University Chile Expedition 1948 - 49. 29. The Crustacea Brachyura of Chile. Lund Univ. Arsskr., N. F. 2, 53(7), 3-127.

Gorny, M. - 1999. The biogeography and ecology of the Southern Ocean decapod fauna. Sci. Mar., 63(Supl. 1): 367-382. 
Gorny, M., W.E. Arntz, A. Clarke and D. J. Gore. - 1992: Reproductive biology of caridean decapods from the Weddell Sea. Polar Biol., 12, 111-120.

Gutt, J., M. Gorny and W. Arntz. - 1991. Spatial distribution of Antarctic shrimps (Crustacea: Decapoda) by underwater photography. Antarct. Sci., 3: 363-369.

Gutt, J. and T. Schickan. - 1996. Epibenthic communities analysed by underwater camera. In: W. Arntz and M. Gorny (eds.), Cruise report of the Joint Chilean-German-Italian Magellan "Victor Hensen" Campaign in 1994. Ber. Polarforsch., 190: 35-41.

Haig, J. - 1955. Reports of the Lund University Chile Expedition 1948 - 49. 20. The Crustacea Anomura of Chile. Lund Univ. Arsskr., N. F. 2, 51(12), 1-68.

Holthuis, L. B. - 1952. Reports of the Lund University Chile Expedition 1948 - 49. 5. The Crustacea Decapoda Macrura of Chile. Lunds Univ. Årsskr., N.F. 2, 47(10): 1-109 .

Kirkwood, J.M. - 1984. A guide to the Decapoda of the Southern Ocean. ANARE Res. Notes, 11: 1-47.

Klages, M., J. Gutt, A. Starmans and T. Bruns. - 1995. Stone crabs close to the Antarctic continent: Lithodes murrayi Henderson, 1888 (Crustacea; Decapoda; Anomura) off Peter I. Island (68 ${ }^{\circ}$ 51'S, 90 51'W). Polar Biol., 15: 73-75.

Mutschke, E. and M. Gorny. - 1999. The benthic decapod fauna in the channels and fjords along the South Patagonian Icefield, Southern Chile. Sci. Mar., 63(Supl. 1): 315-319.

Ramos, A. - 1999. The macrozoobenthos of the Scotia Arc archipelagos. Sci. Mar. 63(Supl. 1): 171-182.

Retamal, M.A. - 1973. Contribución al conocimiento de los crustáceos decápodos de la Región Magallánica. Gayana (Zool.), 29: 1-24.

Retamal, M.A. - 1974. Contribución al conocimiento de los crustáceos decápodos de la Región Magallánica. Gayana (Zool.), 31: 3-23.
Retamal, M.A. - 1992. Los Lithodidae chilenos. Ans. Inst. Pat. Ser. Cs. Nts. 21: 111-129.

Retamal, M.A. and R. Soto. - 1993. Crustáceos decápodos abisales de la zona Iquique-Arica. Estud. Oceanol., 12: 1-8.

Retamal, M.A. and R. Soto. - 1995. Primer registro de Psathyrocaris fragilis Wood-Mason, 1893 en aguas chilenas (Decapoda - Pasiphaeidae). Gayana (Zool.), 59: 117-118.

Tiefenbacher, L. - 1990a. Eualus kinzeri, a new hippolytid species from the Weddell Sea (Antarctica) (Crustacea: Decapoda: Natantia). Spixiana, 13: 117-120.

Tiefenbacher, L. - 1990b. Beiträge zur Taxonomie von Nematocarcinus longirostris Bate, 1888 und Nematocarcinus lanceopes Bate, 1888, neu für die westliche Antarktis. Spixiana, 13: 229-235.

Tiefenbacher, L. - 1994. Decapode Crustaceen aus westantarktischen Gewässern gesammelt von der R.V. "John Biscoe", Reise 11. Spixiana, 17 (1): 13-19.

Vinuesa, J.H. - 1977. Aportes al conocimiento de los crustáceos decápodos de Tierra del Fuego con algunas observaciones zoogeográficas. Physis (Sec. A), 36 (92): 9-19.

Voss, J. - 1988. Zoogeographie und Gemeinschaftsanalyse des Makrozoobenthos der Weddellsee (Antarktis). Ber. Polarforsch., 45: 1-144.

Wehrtmann, I.S. and A. Carvacho. - 1997. New records and distribution ranges of shrimps (Crustacea: Decapoda: Penaeoidea and Caridea) in Chilean waters. Proc. Biol. Soc. Washington, 110(1): 49-57.

Zarenkov, N.A. - 1970. Crustacean Decapoda collected by the Soviet Antarctic expeditions in the Antarctic and antiboreal regions. Biol. Rep. Soviet Antarct. Exped. (1955-1958), 4: 153-201.

Zinsmeister, W.J. and R.M. Feldmann. - 1984. Cenozoic high latitude heterochroneity of southern hemisphere marine faunas. Science, 224: 281-283. 